\title{
A pediatric case of disseminated cystic echinococcosis
}

Authors: Alexandra Poparlan-Constantin ${ }^{1}$, Irina Dijmarescu ${ }^{1}$, Andreia Florina Nita ${ }^{1,2}$, Andreea Girbea ${ }^{1}$, Daniela Pacurar $^{1,2}$ 1."Grigore Alexandrescu" Emergency Children's Hospital, Bucharest, Romania

\section{Background:} 2."Carol Davila" University of Medicine and Pharmacy, Bucharest, Romania

- Echinococcus granulosus eggs' ingestion (found in contaminated soil, food or water) leads to the parasitosis known as echinococcosis

- Parasite enters portal circulation from the upper intestine - develops into cysts in the lungs (most common site in children), liver and other organs

Case report: 16 years old male patient; illiterate parent, from rural area

\section{October 2012 (11 years old) - Surgical Department}

Anaphylactic shock due to the rupture of a hydatid cyst in his right lung Cyst was surgically removed, together with the middle lobe

Albendazole treatment was recommended - not regularly administered

January 2017 (16 years old) - Surgical Department

-Admitted for reevaluation

-Chest $x$-ray - multiple cysts of the right hemi thorax.

- Abdominal ultrasound - multiple different size cystic lesions of the liver and subcutaneous tissue, some of them with daughter vesicles inside - Cranial, thoracic and abdominal CT confirmed - multiple hydatid cysts localized in the pulmonary parenchyma, pleura, liver and subcutaneous tissue. DISSEMINATED HYDATIDOSIS - recommended to continue the antiparasitic therapy with Albendazole

\section{March 2017 - Pediatrics Department}

\section{Presenting complaint: right side lumbar pain}

- Physical examination: surgical scar on the right hemi thorax; on the right posterior axillary line a palpable, mobile, painless mass (Fig. 1)

-Chest $x$-ray - multiple well-defined round homogeneous opacities in the right hemi thorax and ascended right hemidiaphragm due to middle lobectomy (Fig. 2)

-Abdominal and soft tissue ultrasound - multiple hydatid cysts, some of them with daughter vesicles inside, located in the right liver lobe, and the soft tissue in the right laterothoracic region

-Thorax CT - multiple cysts in the mediastinum, pulmonary parenchyma and pleura (Fig. 3) - Abdominal CT - multiple hydatid cysts in the right liver lobe, peripherally located, parahepatic cysts and also a cyst located in the right parietal subcutaneous tissue (Fig. 4)

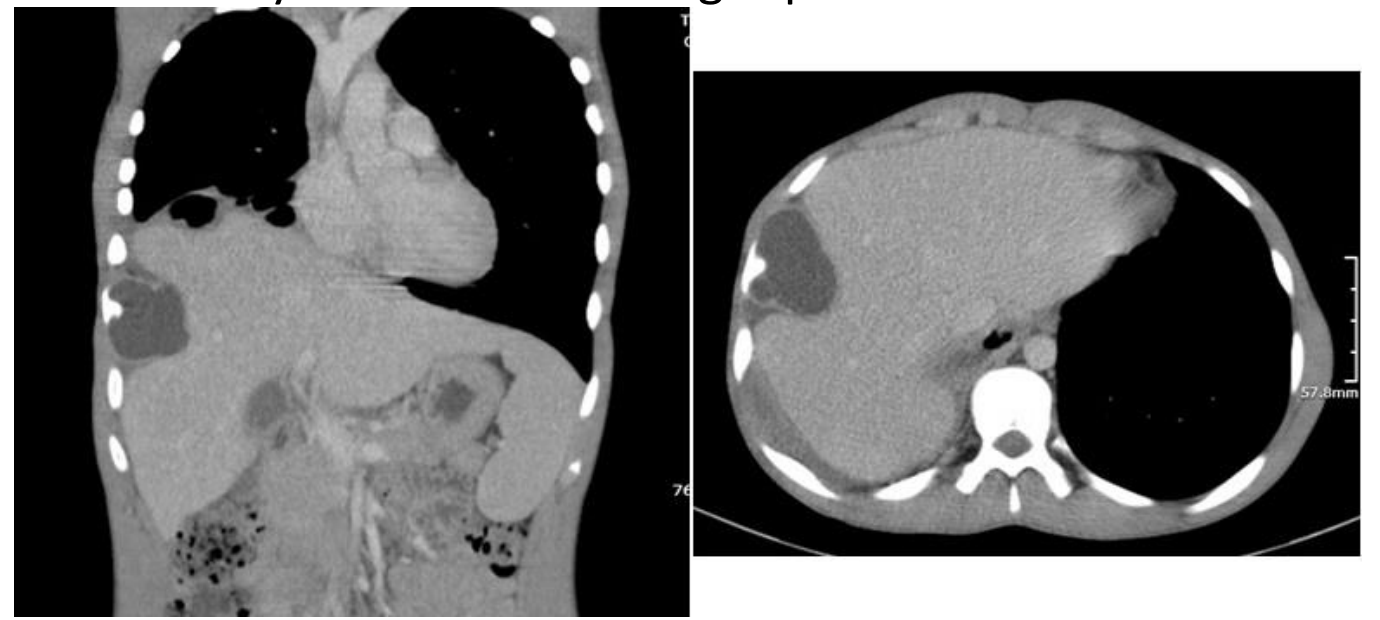

Fig. 4. Abdominal CT - hypodense hypoenhancing fluid content lesion with thin enhancing wall, multilobulated, localized inter-hepato-parietal with mass effect on the hepatic capsule

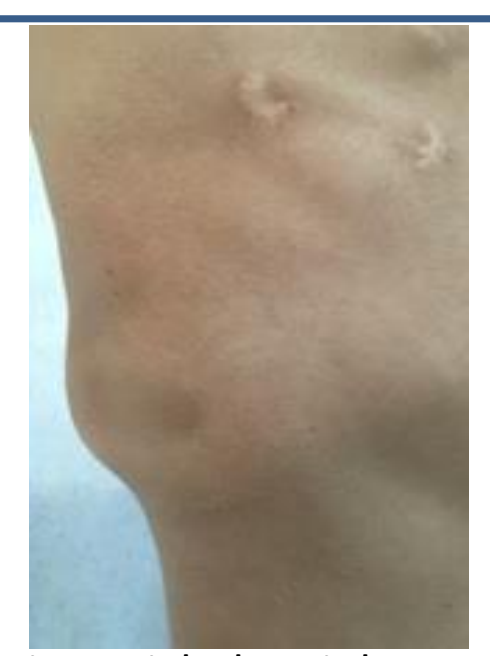

Fig 1. Right hemi thorax surgical scar. Mobile, painless mass on the posterior axillary line.

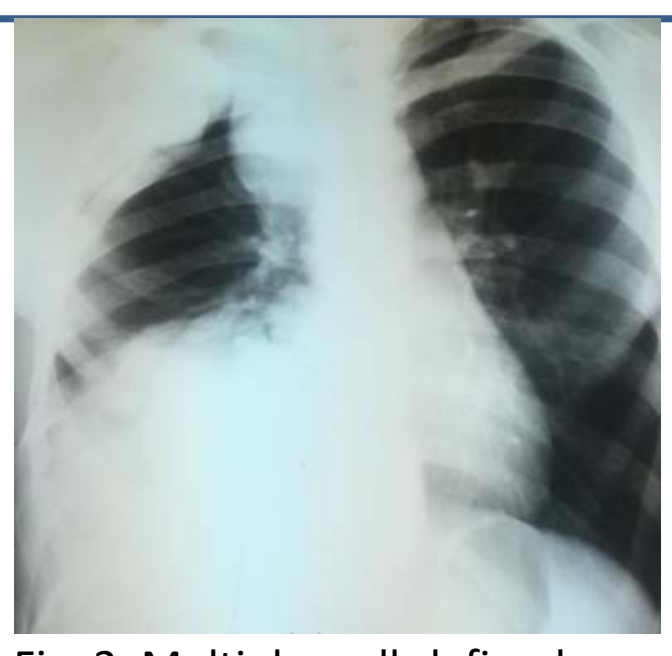

Fig. 2. Multiple well-defined round homogeneous opacities in the right hemi thorax; ascended right hemidiaphragm

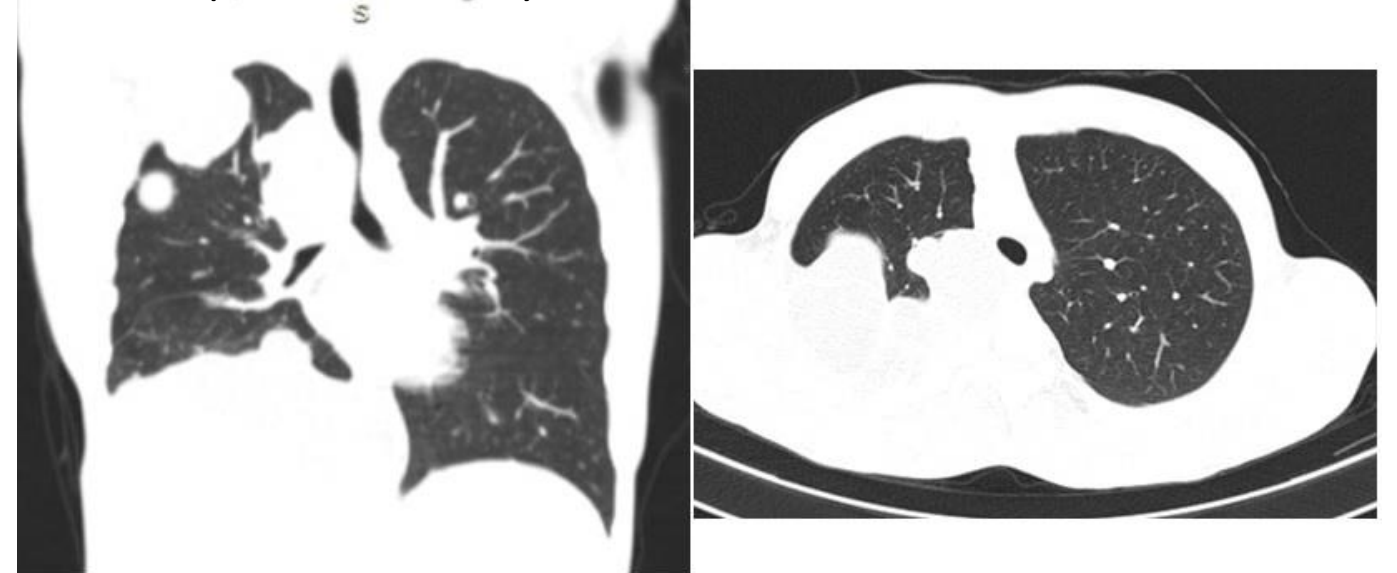

Fig. 3. Thorax CT - round macronodular well defined lesions involving the upper right lobe and inferior lobe showing a mass efect on the trachea and right main bronchi.

\section{Parasitological consult:}

-antiparasitic treatment (Albendazole 200mg every 8 hours)

- Evacuation of the pulmonary hydatid cysts as a first step,

following with the management of the abdominal ones

Reffered to the Surgical team

Lost from follow-up (did not return for scheduled intervention)

\section{September 2017 - Pediatrics Department}

-Involved in a car accident

-Subsequently: fever, severe

persistent spasmodic cough

- Chest X-Ray, Thorax CT: multiple

mixt lesions with hydroaeric levels in

the right hemithorax (Fig. 5, Fig. 6)

-MULTIPLE COMPLICATED

PULMONARY HYDATID CYSTS

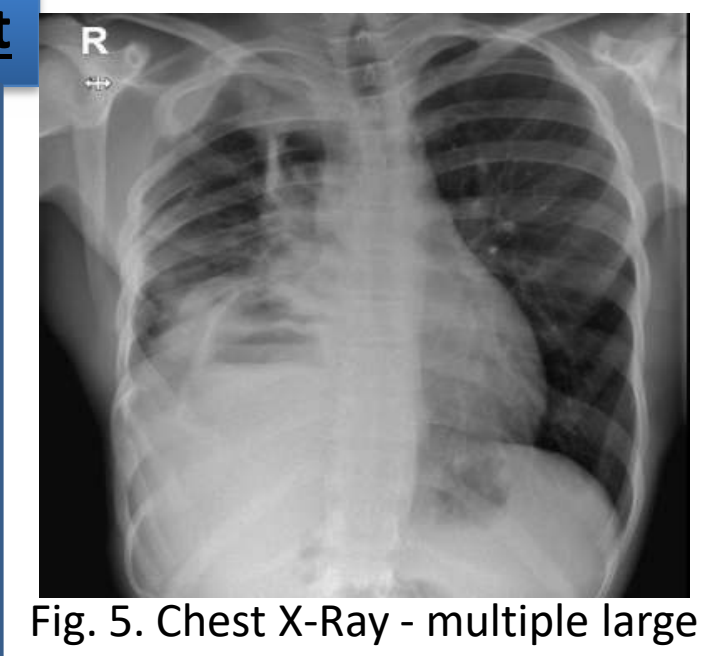

Fig. 5. Chest X-Ray - multiple large

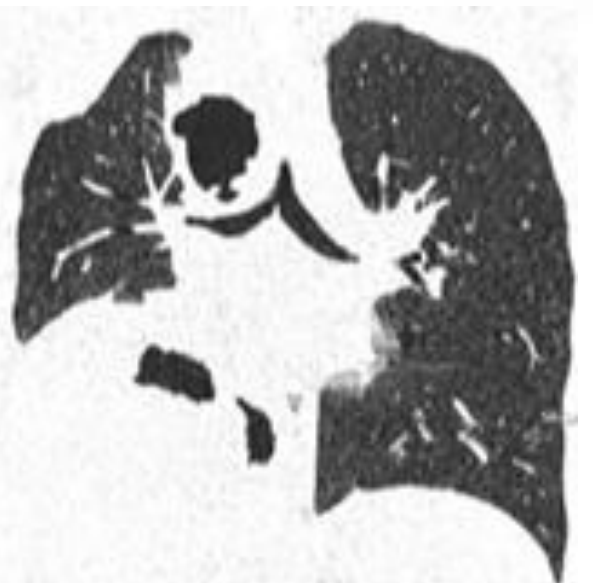

Fig. 6. Thorax CT - multiple cavities with fluid content

\section{Background:}

-The authors presented the case of an adolescent with a potentially curable parasitic infection diagnosed in childhood which became untreatable due to non-medical factors

-The patient originated from illiterate parents and had six other siblings. They were animal breeders (horses) - the child did not attend school and was frequently in the proximity of animals

-The evolution of the patient was severely influenced by the socio-economic level of the family which resulted in lack of compliance to the treatment and nonattendance to follow-up clinics 